\title{
Analytic free-form lens design for imaging applications with high aspect ratio
}

\author{
Fabian Duerr,,${ }^{*} a$ Pablo Benítez, ${ }^{b, c}$ Juan Carlos Miñano, ${ }^{b, c}$ Youri Meuret ${ }^{a}$ and Hugo \\ Thienpont $^{a}$ \\ ${ }^{a}$ Vrije Universiteit Brussel, Faculty of Engineering Sciences, Brussels Photonics Team \\ B-PHOT, TONA-FirW, Pleinlaan 2, 1050 Brussels, Belgium \\ ${ }^{b}$ CeDInt, Universidad Politécnica de Madrid (UPM), Campus de Montegancedo, 28223 \\ Pozuelo de Alarcón, Spain \\ ${ }^{c}$ Light Prescriptions Innovators, 16662 Hale Ave., Irvine, CA USA 92606
}

\begin{abstract}
A new three-dimensional analytic optics design method is presented that enables the coupling of three ray sets with only two free-form lens surfaces. Closely related to the Simultaneous Multiple Surface method in three dimensions (SMS3D), it is derived directly from Fermat's principle, leading to multiple sets of functional differential equations. The general solution of these equations makes it possible to calculate more than 80 coefficients for each implicit surface function. Ray tracing simulations of these free-form lenses demonstrate superior imaging performance for applications with high aspect ratio, compared to conventional rotational symmetric systems.
\end{abstract}

Keywords: Mathematical methods in physics, geometric optical design and lens design, free-form optics

\section{INTRODUCTION}

In optics, a distinction is made between imaging (image-forming) and non-imaging optical systems. Non-imaging optics is concerned with the efficient transfer of light radiation between a source and a target. Unlike traditional imaging optics, instead of an image of the source, the optics produces a prescribed illuminance (or irradiance) pattern on the receiver. ${ }^{1}$ This inherent difference is also reflected in the choice of optical design methods.

Two main problems of non-imaging optics are efficient designs for illumination and solar concentration applications. Most non-imaging optics designs typically use few optical surfaces (often not more than two); advanced free-form optics design methods and solutions evolved over time, both for illumination applications (see, for example $^{2-5}$ ), and in concentration photovoltaics ${ }^{6,7}$ using the Simultaneous Multiple Surfaces (SMS) design method. The limited number of surfaces makes it possible to efficiently design highly aspheric or non-rotational symmetric free-form surfaces to address the system specifications.

Originating from non-imaging optics design, a particular formulation of the SMS2D design method for imaging systems comprises perfect imaging of $N$ ray sets at the correspondent $N$ image points in a plane. ${ }^{8}$ It offers the flexibility to choose the ray sets and their associated image points and design rotational symmetric optics for imaging applications. ${ }^{9-11}$ Recent years have also seen a raising interest in free-form optics for off-axis imaging designs, as well as the extension of aberration theory in imaging systems without rotational symmetry. ${ }^{12-18}$

In case of on-axis imaging, rotational symmetric optics is omnipresent as it greatly simplifies the design process and analysis of optical systems and the manufacturing process. The rotational symmetry of the optical system makes it the best solution for design problems where the object and image are rotational symmetric with respect to the optical axis. In practice, this design approach is also adequate for systems with rectangular field of view and rectangular image receivers characterized by moderate aspect ratios. However, the more the field of view deviates from the rotational symmetric configuration, the more interesting becomes the question of how non-rotational symmetric optics can help to provide tailor-made solutions with increased overall imaging performance.

To illustrate this potential for imaging applications with high aspect ratio, the imaging performance of a rotational symmetric and a free-form (no rotational symmetry) single thick lens are compared in this work.

\footnotetext{
*E-mail correspondence: fduerr@b-phot.org; Telephone: +32 (0)2 6293570
} 
The design of the free-form lens is based on a new analytic optics design method, closely related to the Simultaneous Multiple Surface method. The SMS design method enables the perfect imaging of two ray sets with two aspheric lens profiles in two dimensions, and the perfect imaging of two ray sets with two free-form surfaces in three dimensions. ${ }^{19}$ In general, SMS surfaces are piecewise curves made of several portions of generalized Cartesian ovals that map initial ray sets to final ray sets. SMS optics are calculated by applying a constant optical path length for each coupled ray set. In case of two optical surfaces, two ray sets defined by design angles of opposite sign can be perfectly imaged both in two and in three dimensions. In case of optical systems designed for wide field of view and with at least one surface far from the aperture stop, the new analytic lens design method provides a way to image more than two ray sets with only two lens surfaces. However, this can only be achieved if different ray sets use different portions of the lens surfaces. Optical systems, where different incident directions use different portions of lens' surfaces, are widely known. Field-flattener lenses are used in binocular designs and in astronomic telescopes to improve edge sharpness and lower distortion. Aperture stops in imaging systems often target the same objective.

For optical systems designed for a wide field of view and with clearly separated optical surfaces, the new lens design method discussed in this paper will allow the perfect imaging of an additional on-axis (parallel to the optical axis) ray set to the correspondent image point both in two ${ }^{20}$ and three dimensions. ${ }^{21}$

One explicit two-dimensional solution of such lens profiles, which perfectly focus three sets of parallel rays with incident angles $-12^{\circ}, 0^{\circ}$ and $12^{\circ}$ onto three image points is shown in Fig. 1(a). Based on the two-dimensional lens profiles, there are different possibilities to construct three-dimensional lens surfaces. The most obvious choice is a rotational symmetric lens which perfectly image parallel rays with incident angle $0^{\circ}$, as it is shown in Fig. 1(b). The two-dimensional lens profiles (indicated by solid lines) are rotated about the optical axis. Fig. 1(c) shows a free-form analytic solution, perfectly imaging three sets of parallel rays with incident angles $-12^{\circ}, 0^{\circ}$ and $12^{\circ}$ onto three points. Its cross section in $x$-z-plane (the solid lines in Fig. $1(\mathrm{c})$ ) is given by the two-dimensional design method and provides a partial solution (depending on $x$-coordinate only) to the full three-dimensional problem.

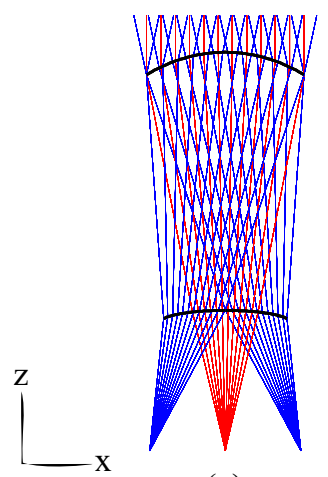

(a)

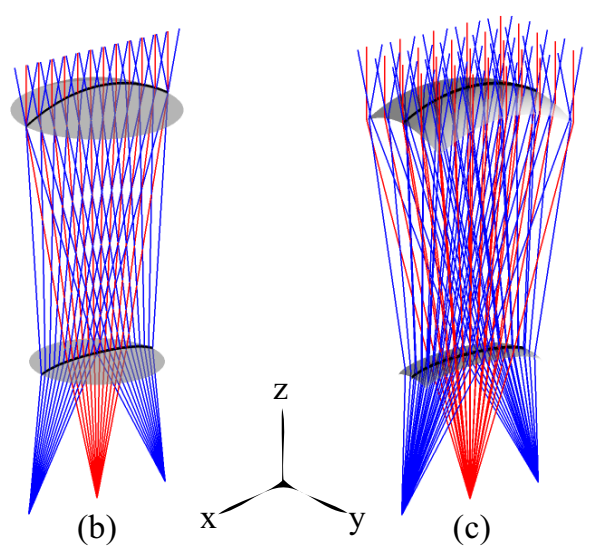

(b) (c)

Figure 1. (a) Lens profiles calculated using the two-dimensional analytic design method to perfectly image three rays sets; (b) rotational symmetric lens constructed from such two-dimensional lens profiles; (c) free-form lens design to perfectly image three ray sets in three dimensions

The analytic lens design method and its main characteristics will be explained and summarized in Sec. 2. It is derived directly from Fermat's principle, leading to multiple sets of functional differential equations. Their transformation into linear systems of equations allows the successive calculation of the analytic Taylor series coefficients in two variables up to an arbitrary order. In Sec. 3, the imaging performance in the limit of an infinite aspect ratio will be compared for a rotational symmetric and a free-form lens which is derived from the high-order Taylor polynomials in two variables. In this specific case, the field of view consists of coplanar parallel directional vectors (objects at infinity) and the correspondent image reduces to a line. This analysis demonstrates the enhanced imaging properties of the non-rotational symmetric free-form lens. As a generalization, the object space will be extended to a field of view with very high but finite aspect ratio in Sec. 4. The performed ray tracing analysis shows that the free-form lens still provides superior imaging performance for applications with 
high aspect ratio, compared to conventional rotational symmetric systems. The final Sec. 5 provides conclusions and possible future developments.

\section{SUMMARY OF THE ANALYTIC FREE-FORM LENS DESIGN METHOD}

The convergence points for one on-axis and one off-axis ray set will form the starting point for all further considerations. An in detail discussion on their existence can be found in. ${ }^{20}$ The implementation of convergence points was used for the first time to design free-form V-groove reflectors. ${ }^{22,23}$ In our design approach, they are characterized by on- and off-axis rays sharing identical points and normal vectors on each lens surface. All necessary initial values are defined as shown in Fig. 2(a).

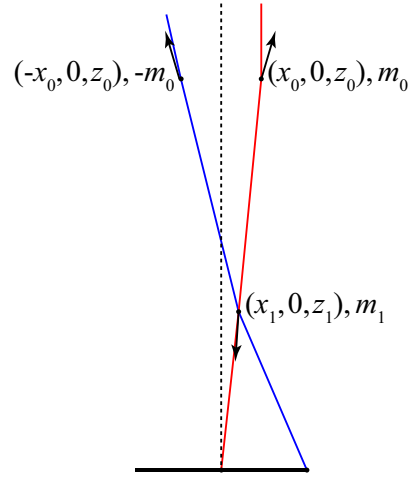

(a) $(0,0, d)(r, 0, d)$

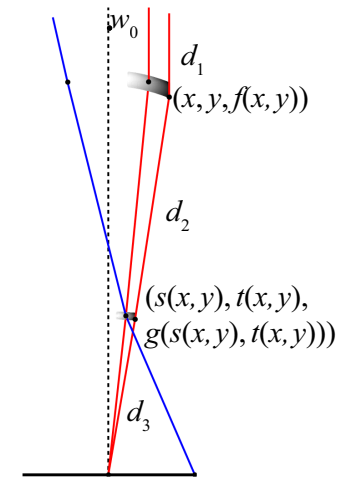

(b)

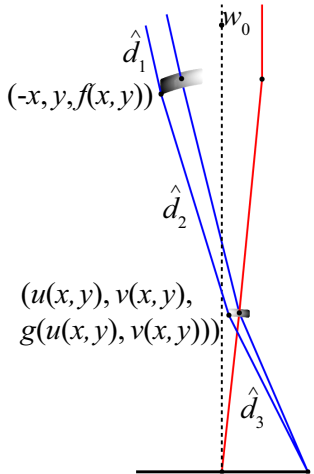

(c)

Figure 2. Introduction of all necessary initial values and functions to derive the conditional equations from Fermat's principle in three dimensions

As one main result it has been shown that the design problem can be fully described by two variables only, the slopes $m_{0}$ and $m_{1}$ at the convergence points. ${ }^{20,21}$ The convergence point on the upper surface has the point coordinates $\left( \pm x_{0}, y_{0}, z_{0}\right)$ with $y_{0}=0$ due to the overall lens' mirror symmetry with respect to the $x$ - $z$ - and $y$ - $z$ plane. Therefore, the normal vector at the first convergence point is completely described by the single variable $m_{0}$. The intersection of the refracted on-axis ray through $\left(x_{0}, 0, z_{0}\right)$ and the refracted off-axis ray through the mirrored convergence point $\left(-x_{0}, 0, z_{0}\right)$ determines the coordinates $\left(x_{1}, 0, z_{1}\right)$ of the second convergence point. The normal vector at the second convergence point is also described by a the single variable $m_{1}$. Further refractions at the second convergence point result in the focus positions $(0,0, d)$ and $(r, 0, d)$. The initial ray construction lies within the sectional plane $y=0$ of the lens and corresponds exactly to the two-dimensional construction.

Next, all necessary functions describing the optical system have to be introduced. Therefore, two explicit surface functions $z=f(x, y)$ and $z=g(x, y)$ are defined to describe the lens surfaces. To completely describe the optical paths of rays passing through the lens surfaces, it is necessary to introduce additional mapping functions $s(x, y)$ and $t(x, y)$ for on-, and $u(x, y)$ and $v(x, y)$ for off-axis rays, and for $x$-direction and $y$-direction, respectively.

Fig. 2(b) shows an on-axis ray passing through an arbitrary point $\overrightarrow{p_{1}}=(x, y, f(x, y))$ on the upper lens surface which is then refracted in $\overrightarrow{p_{2}}=(s(x, y), t(x, y), g(s(x, y), t(x, y)))$ towards the focal point $\overrightarrow{p_{3}}=(0,0, d)$. Similarly, Fig. 2(c) shows an off-axis ray passing through an arbitrary point $\hat{\overrightarrow{p_{1}}}=(-x, y, f(x, y))$ on the upper lens surface which is then refracted in $\hat{\overrightarrow{p_{2}}}=(u(x, y), v(x, y), g(u(x, y), v(x, y)))$ towards the focal point $\hat{\overrightarrow{p_{3}}}=(r, 0, d)$. All optical path lengths can be expressed in sections using vector geometry as

$$
d_{1}=\vec{v}_{0} \cdot\left(\overrightarrow{p_{1}}-\vec{w}_{0}\right), \quad d_{2}=n_{2}\left|\overrightarrow{p_{2}}-\overrightarrow{p_{1}}\right|, \quad d_{3}=\left|\overrightarrow{p_{3}}-\overrightarrow{p_{2}}\right|
$$

for on axis rays, and as

$$
\hat{d}_{1}=\vec{v}_{1} \cdot\left(\hat{\overrightarrow{p_{1}}}-\vec{w}_{0}\right), \quad \quad \hat{d}_{2}=n_{2}\left|\hat{\overrightarrow{p_{2}}}-\hat{\overrightarrow{p_{1}}}\right|, \quad \hat{d}_{3}=\left|\hat{\overrightarrow{p_{3}}}-\hat{\overrightarrow{p_{2}}}\right|
$$

for off-axis rays. The vectors $\vec{v}_{0}$ and $\vec{v}_{1}$ denote the directional vectors for on- and off-axis ray sets, respectively. The position vector $\vec{w}_{0}$ denotes an arbitrary but fixed point on both plane wave-fronts and $n_{2}$ denotes the 
refractive index of the lens. Fermat's principle is now applied to calculate all terms for $x$ - and $y$-coordinate dependency. The conditional equations are defined as

$$
D_{1}=\frac{\partial}{\partial x}\left(d_{1}+d_{2}\right)=0, \quad D_{2}=\frac{\partial}{\partial y}\left(d_{1}+d_{2}\right)=0, \quad D_{3}=\frac{\partial}{\partial s}\left(d_{2}+d_{3}\right)=0, \quad D_{4}=\frac{\partial}{\partial t}\left(d_{2}+d_{3}\right)=0
$$

for on-axis, and as

$$
D_{5}=\frac{\partial}{\partial x}\left(\hat{d}_{1}+\hat{d}_{2}\right)=0, \quad D_{6}=\frac{\partial}{\partial y}\left(\hat{d}_{1}+\hat{d}_{2}\right)=0, \quad D_{7}=\frac{\partial}{\partial u}\left(\hat{d}_{2}+\hat{d}_{3}\right)=0, \quad D_{8}=\frac{\partial}{\partial v}\left(\hat{d}_{2}+\hat{d}_{3}\right)=0
$$

for off-axis rays. The lens design as introduced in Fig. 2 is then fully described by the eight functional differential equations (3) and (4) for six unknown surface functions $f(x, y), g(x, y), s(x, y), t(x, y), u(x, y)$ and $v(x, y)$. To our knowledge, the existence and uniqueness of solutions to similar systems of functional differential equations in two variables have not been discussed in detail nor proven up to now.

Supposing that there exists an analytic and smooth solution $(f, g, s, t, u, v)$ to the functional differential equations (3) and (4), Taylors theorem implies that the functions must be infinitely differentiable and have a power-series representation in two variables. Thus the six functions can be given by power-series

$$
\begin{aligned}
f(x, y) & =\sum_{i=0}^{\infty} \sum_{j=0}^{\infty} f_{i, j}\left(x-x_{0}\right)^{i} y^{2 j}, & g(x, y) & =\sum_{i=0}^{\infty} \sum_{j=0}^{\infty} g_{i, j}\left(x-x_{1}\right)^{i} y^{2 j} \\
s(x, y) & =\sum_{i=0}^{\infty} \sum_{j=0}^{\infty} s_{i, j}\left(x-x_{0}\right)^{i} y^{2 j}, & u(x, y) & =\sum_{i=0}^{\infty} \sum_{j=0}^{\infty} u_{i, j}\left(x-x_{0}\right)^{i} y^{2 j} \\
t(x, y) & =\sum_{i=0}^{\infty} \sum_{j=1}^{\infty} t_{i, j}\left(x-x_{0}\right)^{i} y^{(2 j-1)}, & v(x, y) & =\sum_{i=0}^{\infty} \sum_{j=1}^{\infty} v_{i, j}\left(x-x_{0}\right)^{i} y^{(2 j-1)}
\end{aligned}
$$

centered at the convergence points $\left(x_{0}, 0, z_{0}\right)$ and $\left(x_{1}, 0, z_{1}\right)$, respectively. The exponents for $y$-coordinate take already into account that all functions are either even (provided by $2 j$ ) or odd (provided by $2 j-1$ ) in $y$-direction. This symmetry follows immediately from the later introduced equations (10) and (11). The linear systems of equations for the correspondent Taylor series coefficients have null vectors as only possible solutions and are therefore discarded already in the Taylor series. The in Fig. 2(a) introduced and in equations (5)-(7) assigned initial conditions

$$
\begin{aligned}
& f\left(x_{0}, 0\right)=z_{0},\left.\quad \partial_{x} f(x, y)\right|_{\left(x_{0}, 0\right)}=m_{0},\left.\quad \partial_{y} f(x, y)\right|_{\left(x_{0}, 0\right)}=0 \\
& g\left(x_{1}, 0\right)=z_{1},\left.\quad \partial_{x} g(x, y)\right|_{\left(x_{1}, 0\right)}=m_{1},\left.\quad \partial_{y} g(x, y)\right|_{\left(x_{1}, 0\right)}=0
\end{aligned}
$$

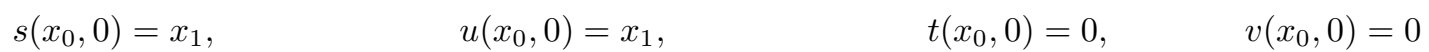

then satisfy the conditional equations $D_{i}=0$ for $i=1 . .8$ and provide general solutions for the initial Taylor series coefficients. The overall solution can be calculated by solving four equations

$$
\lim _{x \rightarrow x_{0}} \lim _{y \rightarrow 0} \frac{\partial^{n}}{\partial x^{n}} \frac{\partial^{m}}{\partial y^{m}} D_{i}=0 \quad(i=1,3,5,7), \quad\left\{n \in \mathbb{N}_{1}, m=0\right\}
$$

for $x$-coordinate dependency, four equations

$$
\lim _{x \rightarrow x_{0}} \lim _{y \rightarrow 0} \frac{\partial^{n}}{\partial x^{n}} \frac{\partial^{m}}{\partial y^{m}} D_{i}=0 \quad(i=2,4,6,8), \quad\{n=0, m \in \mathbb{N} \mid \text { odd number } m\}
$$

for $y$-coordinate dependency, and six equations

$$
\begin{array}{ll}
\lim _{x \rightarrow x_{0}} \lim _{y \rightarrow 0} \frac{\partial^{n}}{\partial x^{n}} \frac{\partial^{m}}{\partial y^{m}} D_{i}=0 \quad(i=2,4,6,8), \quad\left\{n \in \mathbb{N}_{1}, m \in \mathbb{N} \mid \text { odd number } m\right\} \\
\lim _{x \rightarrow x_{0}} \lim _{y \rightarrow 0} \frac{\partial^{n-1}}{\partial x^{n-1}} \frac{\partial^{m+1}}{\partial y^{m+1}} D_{i}=0 \quad(i=3,7), \quad\left\{n \in \mathbb{N}_{1}, m \in \mathbb{N} \mid \text { odd number } m\right\}
\end{array}
$$

for mixed terms with $x$-y-coordinate dependency. To derive the solution scheme, it is useful to introduce an ordinal number $o=m+n$. There are two cases needed to be solved: 
1. For $o=1$, the sets of equations (9) and (10) each result in four nonlinear algebraic equations for Taylor series coefficients $f_{2,0}, g_{2,0}, s_{1,0}$ and $u_{1,0}$, and for $f_{0,2}, g_{0,2}, t_{0,1}$ and $v_{0,1}$. These equations have each two general solutions, where one solution can be discarded as non-physical. The remaining unique solutions can be expressed as functions of the initial, already known Taylor coefficients.

2. For $o \geq 2$, The sets of equations (9), (10) and (11) result in three systems of linear equations for particular Taylor series coefficients which can be expressed as compact matrix equations as follows

$$
M_{x}\left(\begin{array}{c}
f_{n+1,0} \\
g_{n+1,0} \\
s_{n, 0} \\
u_{n, 0}
\end{array}\right)=\vec{b}^{(n, 0)}, \quad M_{y}\left(\begin{array}{c}
f_{0, m+1} \\
g_{0, m+1} \\
t_{0, m} \\
v_{0, m}
\end{array}\right)=\vec{b}^{(0, m)}, \quad M_{x y}\left(\begin{array}{c}
f_{n, m+1} \\
g_{n, m+1} \\
s_{n-1, m+1} \\
t_{n, m} \\
u_{n-1, m+1} \\
v_{n, m}
\end{array}\right)=\vec{b}^{(n, m)}
$$

for $x$-coordinate dependency, $y$-coordinate dependency and $x$-y-coordinate dependency, respectively. The matrix elements of $M_{x}, M_{y}$ and $M_{x y}$ consist of mathematical expressions which depend on Taylor series coefficients calculated for the initial conditions. The needed vector elements of $\vec{b}^{(n, m)}$ are mathematical expressions only dependent on previously calculated Taylor series coefficients for $o=2,3, \ldots,(n+m-1)$ and can be calculated for each ordinal number $o=2,3, .$. in ascending order and for all possible combinations of $n$ and $m$ from equations (9)-(11). For known matrices $M_{x}, M_{y}$ and $M_{x y}$ and vectors $\vec{b}^{(n, m)}$, the Taylor series coefficients can be calculated by solving the linear systems of equations (12).

The presented solution provides a scheme to calculate the Taylor polynomial coefficients in equations (5)-(7) up to an arbitrary but finite order. Taylors remainder theorem in two variables provides quantitative estimates on convergence and the approximation error of the functions by its Taylor polynomials. The radii of convergence for the expansions $f(x, y)$ and $g(x, y)$ are very important, as they indicate the maximum aperture that can be achieved for given initial values. The presented calculations are fully implemented in Wolfram Mathematica.

\section{PERFORMANCE EVALUATION FOR LINE IMAGING SYSTEMS}

All calculated mathematical expressions, sorted in the right order, are exported as C++ compatible code and embedded in a MATLAB-compatible mex file library. Once compiled, this library returns the calculated Taylor polynomial coefficients for the lens surfaces $f(x, y)$ and $g(x, y)$ and the mapping functions $s(x, y), t(x, y), u(x, y)$ and $v(x, y)$ for input parameters $\left(\theta, m_{0}, m_{1}, n_{2}\right)$. The Taylor coefficients are calculated up to $o=15$, resulting in a total number of 81 Taylor coefficients for $f(x, y)$ and $g(x, y)$, respectively. This derived general three-dimensional calculation provides solutions for any given (physically meaningful) initial parameter set $\left(\theta, m_{0}, m_{1}, n_{2}\right)$.

For fixed design angle and refractive index, the only remaining free parameters to vary are the slopes $m_{0}$ and $m_{1}$. The lens' smoothness and symmetry additionally requires that the boundary condition $\left.\partial_{x} f(x, y)\right|_{(0,0)}=$ $\left.\partial_{x} g(x, y)\right|_{(0,0)}$ at the optical axis is fulfilled. In previous work, the evaluation of the solution space revealed the wide range of possible pairs of variates $m_{0}$ and $m_{1}$ resulting in different lens configurations ranging from biconvex to meniscus lens shapes, covered by this analytic design method. ${ }^{24}$

In order to compare the line imaging performance of a rotational symmetric lens (see Fig. 1(b)) and a free-form lens (see Fig. 1(c)) solution, the following initial parameter set is selected $\left(11^{\circ},-0.2663,-0.0486,1.5\right)$ for both lens configurations. The ray tracing evaluation in this work is done using Synopsys' Code V. As Code $V$ (version 10.3) does not support the direct implementation of general Taylor polynomials centered at a point $x_{0} \neq 0$, the solutions have to be transformed. The two-dimensional lens profiles are fitted to asphere surfaces (ASP) using an approximation method of least squares in MATLAB. The translated aspheres' coefficients up to $16^{\text {th }}$ order can be directly imported into Code $V$ and used to define the circular rotational symmetric lens. A 3D viewing of ray tracing for the rotational symmetric lens is shown in Fig. 3(a). The three-dimensional free-form lens surfaces are fitted to XY Polynomial (SPS XYP) surfaces using an approximation method of least squares in MATLAB as well. The SPS XYP surface is defined by a series of polynomials (up to the $10^{\text {th }}$ order) added to a base conic and the polynomial is expanded into monomials of $x^{n} y^{m}$, where $(m+n) \leq 10$, a lower but still sufficiently high order compared to the order that the analytic solution provides (backed by the obtained ray 


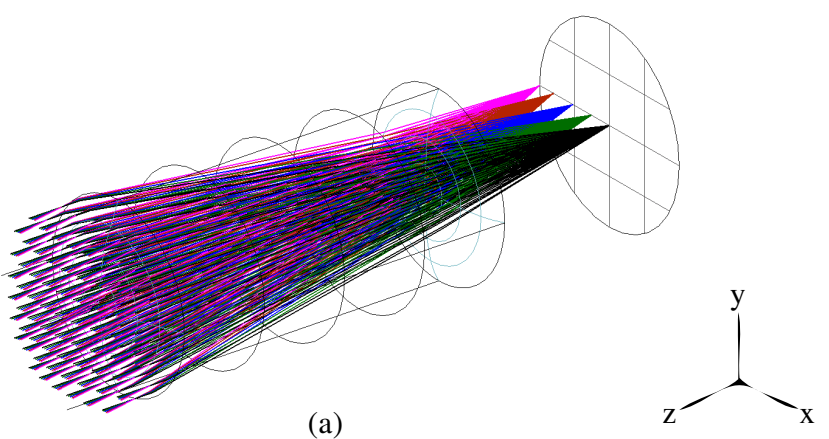

(a)

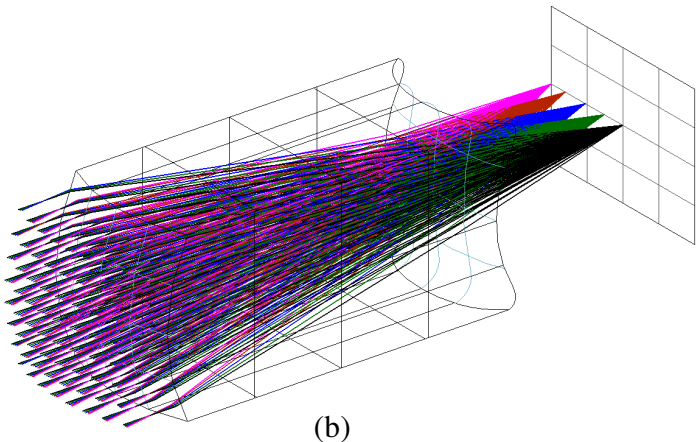

(b)

Figure 3. 3D viewing of ray tracing for the circular rotational symmetric lens (a) and the rectangular free-form lens (b)

tracing results). Due to the lens' symmetry, only about 20 coefficients for each lens surface do not equal zero. A 3D viewing of ray tracing for the free-form lens is shown in Fig. 3(b). The entry aperture diameter of the rotational symmetric lens measures $3.4 \mathrm{~mm}$, the free-form lens has an entry aperture of $2.84 \mathrm{~mm} \times 3.2 \mathrm{~mm}$ to result in identical aperture sizes. The f-number of the rotational symmetric lens is about $\mathrm{f} / 2.7$. The free-form lens' f-numbers are about $\mathrm{f} / 2.8$ in $x$-direction and about $\mathrm{f} / 3.0$ in $y$-direction, respectively. The same aperture sizes of the lower lens surfaces ensure that all rays going through the first lens surface also reach the image plane. To evaluate the line imaging performance of both lenses, the spot diagrams for different field positions along the $x$-axis (see Fig. 3) are shown in Fig. 4 for the rotational symmetric lens (a) and the free-form lens (b). The added

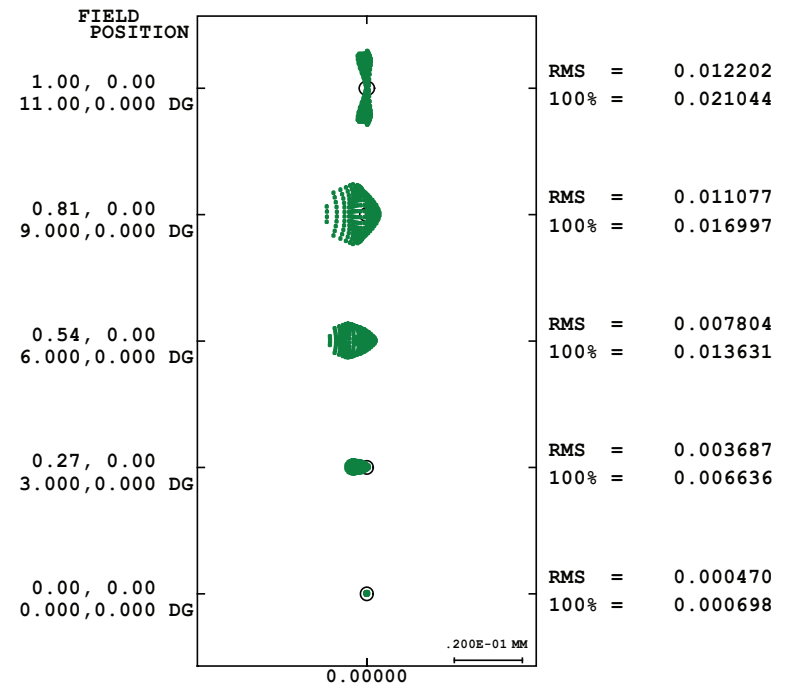

(a)

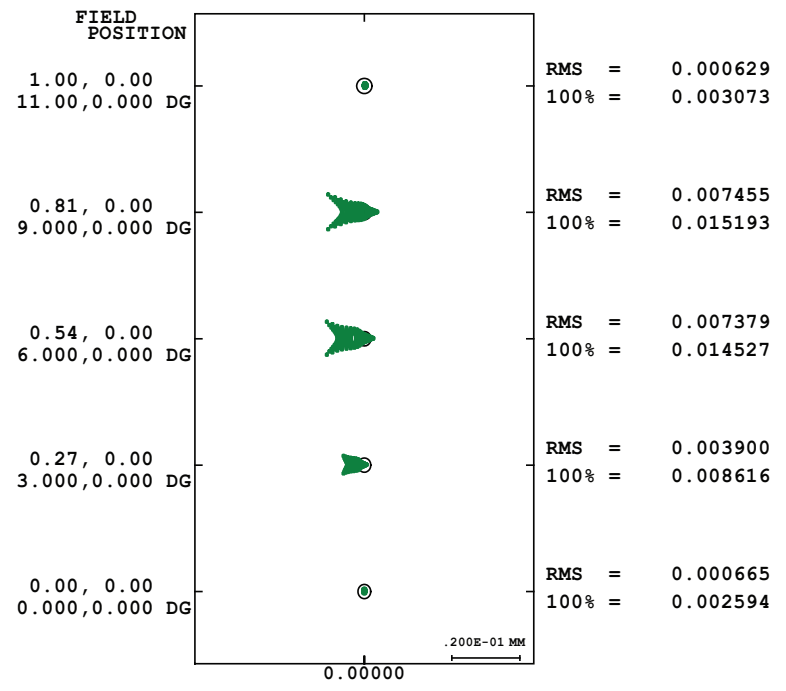

(b)

Figure 4. Spot diagrams for the circular rotational symmetric lens (a) and the rectangular free-form lens (b)

circles to the spot diagrams correspond to the Airy disk diameters (determined from real ray tracing) at the reference wavelength. As expected by the design characteristics, the RMS spot diameters at the design angle $0^{\circ}$ in case of the rotational symmetric lens and at the design angles $0^{\circ}$ and $11^{\circ}$ in case of the free-form lens are close to zero (of the order of 0.5 microns), free from aberrations (like e.g., coma) and lie within the Airy disk diameters. In direct comparison, the analysis for intermediate incident directions demonstrate a considerably better overall line imaging performance for the free-form lens when compared with its rotational symmetric counterpart both for the RMS as well as the $100 \%$ spot diameters. 


\section{PERFORMANCE EVALUATION FOR AN EXTENDED FIELD OF VIEW}

In this section, the performance evaluation is extended to a field of view with very high aspect ratio while the investigated systems remain identical as in Sec. 3. The RMS spot diameter field maps are calculated using Code $V$ for a $26^{\circ} \times 5^{\circ}$ field of view. This chosen field of view appears arbitrary, but ensures for both lens designs that all rays passing through the first lens surface still reach the image plane. Due to the systems' symmetries, only a quadrant of the entire field of view is needed to be analyzed. Fig. 5 shows the contour plots of the RMS spot diameter distributions for the rotational symmetric lens (top) and the free-form lens (bottom).
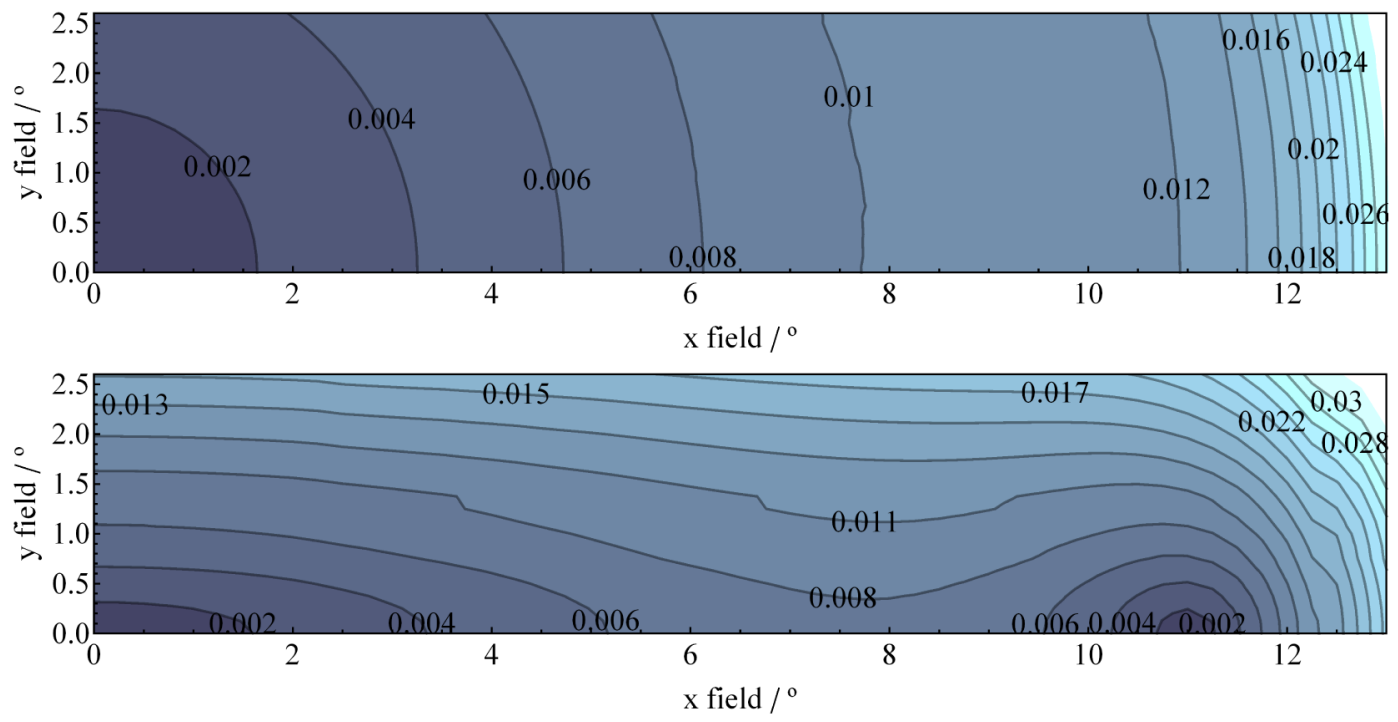

Figure 5. RMS spot diameter distributions for the circular rotational symmetric lens (top) and the rectangular free-form lens (bottom)

The RMS spot diameter distribution of the rotational symmetric lens shares the same symmetry as the optical system. In case of a field of view with very high aspect ratio, the imaging performance hardly varies from the previous line imaging performance and is mainly dominated by the $x$ field dependency of the RMS spot diameters, as the contour lines become more and more vertically aligned with an increasing horizontal field angle.

The situation is quite different in the case of the free-form lens: The contour lines of this RMS spot diameter distribution can essentially be divided into two main regions. Horizontal contour lines for larger $y$ field angles, and (curved) vertical contour lines beyond the off-axis design angle, forming approximately rectangular contour lines for this RMS spot diameter distribution.

As for most direct design methods, an optimization step is typically useful once the initial shape has been derived. Therefore, monochromatic Automatic Design optimization with Transverse Ray Aberration as error function is carried out in Code $V$ to find better solutions for both lens systems for a $24^{\circ} \times 2.4^{\circ}$ (10:1 aspect ratio) field of view. The aspheric and polynomial coefficients of the rotational symmetric lens and the free-form lens are optimized to reduce the RMS spot diameters within the specified field of view. Fig. 6 shows the contour plots of the optimized RMS spot diameter distributions for the rotational symmetric lens (top) and the free-form lens (bottom).

The RMS spot diameter distribution's symmetry of the optimized rotational symmetric lens obviously remains unchanged with its RMS spot diameter values now ranging between 3 and 11 microns, compared to 0.4 and 17 microns in case of the analytic design in the previous section and for the same field of view. The RMS spot diameter distribution of the optimized free-form lens sees a shift and attenuation of the former sharp foci. The RMS spot diameter values range now between 2 and 6 microns, compared to 0.6 and 14 microns in case of the analytic design in the previous section and for the specified field of view. In direct comparison, the overall imaging performance of the optimized free-form lens exceeds the performance of the optimized rotational symmetric lens almost by a factor of two (in terms of maximal RMS spot diameter size) for this field of view with its 10:1 aspect ratio. This result, however, is less about the absolute values itself, it should emphasize the potential use of 

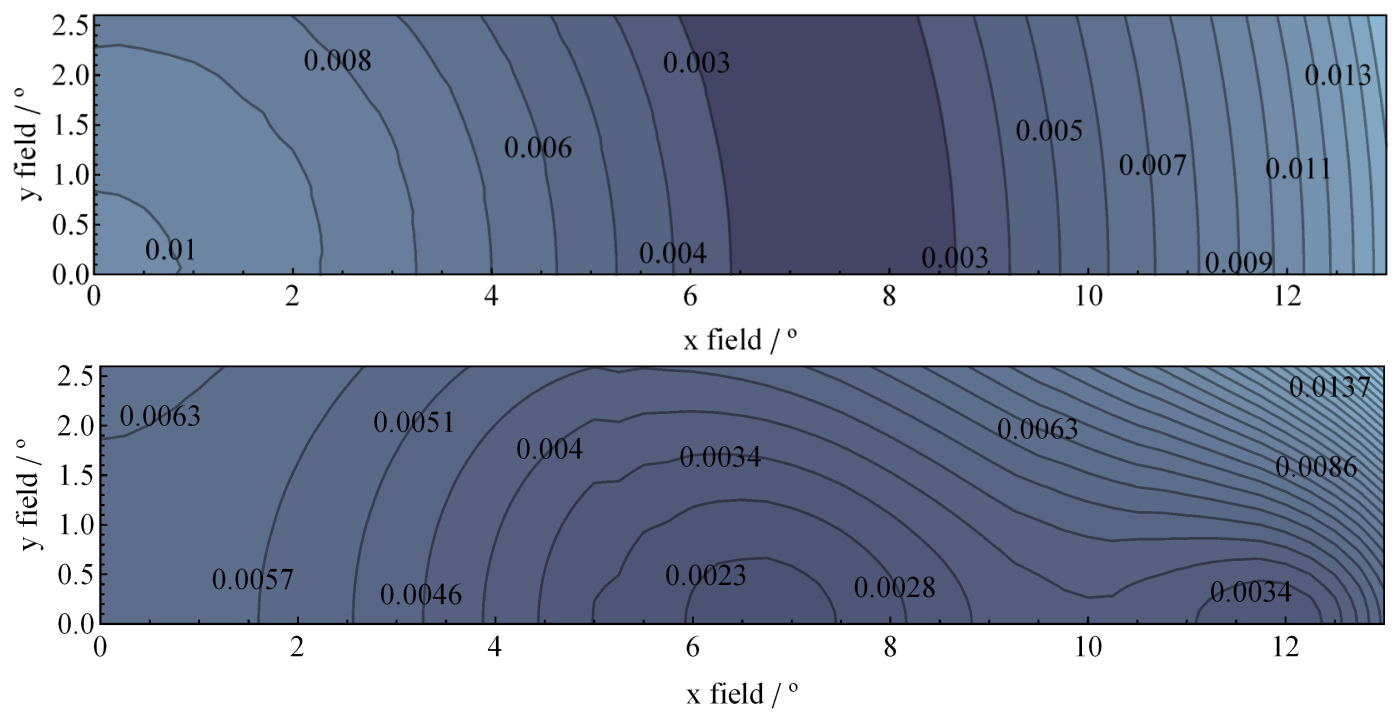

Figure 6. RMS spot diameter distributions for the optimized circular rotational symmetric lens (top) and the rectangular free-form lens (bottom)

free-form optics for on-axis imaging applications where the specified field of view is very far from being circular symmetric. Even for very common and moderate rectangular aspect ratios such as 16:9 HD video standard, 1.85:1 US widescreen cinema standard or 2.39:1 anamorphic widescreen cinema standard, it seems reasonable to assume that the optical system design will also benefit from tailor-made free-form optics. These are possible optical design questions to be addressed in future work. However, the question remains if the potential increase in the image performance justifies the higher manufacturing complexity and cost of free-form optics.

\section{CONCLUSIONS}

Within the scope of this work, a new three-dimensional analytic optics design method has been presented that allows the coupling of three ray sets with only two free-form lens surfaces. The convergence point formalism and Fermat's principle provided the basis for an analytical description enabling the simultaneous and accurate calculation of two free-form lens surfaces up to very high orders. It is particularly notable that this general analytic solution depends only on very few free parameters, ideal for optimization.

The line imaging performance evaluation for calculated lens surfaces given as high order Taylor polynomials of two variables demonstrated the superiority of the free-form lens solution when compared to its conventional rotational symmetric counterpart. For an extended field of view with 10:1 aspect ratio, the overall imaging performance of the optimized free-form lens exceeded the performance of the optimized rotational symmetric lens almost by a factor of two (in terms of maximal RMS spot diameter size).

Future work will further elaborate the development of this new optics design method for both imaging and non-imaging optics applications. This implies that more than two surfaces, different symmetry considerations and more generalized wave-fronts to image objects at a finite distance should be considered.

\section{ACKNOWLEDGMENTS}

Work reported in this paper is supported in part by the Research Foundation - Flanders (FWO-Vlaanderen) that provides a PhD grant (grant number FWOTM510) and provided a grant for a research period at CeDInt, Universidad Politécnica de Madrid (grant number V424711N) for Fabian Duerr, and in part by the IAP BELSPO VI-10, the Industrial Research Funding (IOF), Methusalem, VUB-GOA, and the OZR of the Vrije Universiteit Brussel.

Work is also supported by projects TEC2011-24019 and SEM: TSI-020302-2010-65, funded by the Spanish Ministries MEC and MITYC, respectively. 


\section{REFERENCES}

[1] Chaves, J., [Introduction to nonimaging optics], CRC (2008).

[2] Ries, H. and Muschaweck, J., "Tailored freeform optical surfaces," JOSA A 19(3), 590-595 (2002).

[3] Glimm, T. and Oliker, V., "Optical design of single reflector systems and the Monge-Kantorovich mass transfer problem," J. Math. Sci. 117(3), 4096-4108 (2003).

[4] Oliker, V., "On design of free-form refractive beam shapers, sensitivity to figure error, and convexity of lenses," JOSA A 25(12), 3067-3076 (2008).

[5] Fournier, F., Cassarly, W., and Rolland, J., "Fast freeform reflector generation using source-target maps," Opt. Express 18(5), 5295-5304 (2010).

[6] Hernández, M., Cvetkovic, A., Benítez, P., and Miñano, J. C., "High-performance Köhler concentrators with uniform irradiance on solar cell," Proc. SPIE 7059, 705908 (2008).

[7] Benítez, P., Miñano, J. C., Zamora, P., Mohedano, R., Cvetkovic, A., Buljan, M., Chaves, J., and Hernández, M., "High performance Fresnel-based photovoltaic concentrator," Opt. Express 18, A25-A40 (2010).

[8] Miñano, J. C., Benítez, P., Lin, W., Infante, J. M., Muñoz, F., and Santamaría, A., "An application of the SMS method for imaging designs," Opt. Express 17(26), 24036-24044 (2009).

[9] Infante Herrero, J. M., Muñoz, F., Benítez, P., Miñano, J. C., Wang, L., Vilaplana, J., Biot, G., and de La Fuente, M., "Novel fast catadioptric objective with wide field of view," Proc. SPIE 7787, 778704 (2010).

[10] Lin, W., Benítez, P., Miñano, J. C., Infante, J. M., and Biot, G., "Advances in the SMS design method for imaging optics," Proc. SPIE 8167, 81670M (2011).

[11] Lin, W., Benítez, P., Miñano, J. C., Infante, J. M., Biot, G., and de la Fuente, M., "SMS-based optimization strategy for ultra-compact SWIR telephoto lens design," Opt. Express 20(9), 9726-9735 (2012).

[12] Plummer, W. T., Baker, J. G., and Van Tassell, J., "Photographic optical systems with nonrotational aspheric surfaces," Appl. Opt. 38(16), 3572-3592 (1999).

[13] Nakano, T. and Tamagawa, Y., "Configuration of an off-axis three-mirror system focused on compactness and brightness," Appl. Opt. 44(5), 776-783 (2005).

[14] Cakmakci, O. and Rolland, J., "Design and fabrication of a dual-element off-axis near-eye optical magnifier," Opt. Lett. 32(11), 1363-1365 (2007).

[15] Cakmakci, O., Moore, B., Foroosh, H., and Rolland, J., "Optimal local shape description for rotationally non-symmetric optical surface design and analysis," Opt. Express 16(3), 1583-1589 (2008).

[16] Thompson, K., Schmid, T., Cakmakci, O., and Rolland, J., "Real-ray-based method for locating individual surface aberration field centers in imaging optical systems without rotational symmetry," JOSA A 26(6), 1503-1517 (2009).

[17] Fuerschbach, K., Rolland, J., and Thompson, K., "A new family of optical systems employing $\varphi$-polynomial surfaces," Opt. Express 19(22), 21919-21928 (2011).

[18] Takahashi, K., "Development of ultrawide-angle compact camera using free-form optics," Opt. Rev. 18(1), 55-59 (2011).

[19] Benítez, P., Miñano, J. C., Blen, J., Mohedano, R., Chaves, J., Dross, O., Hernández, M., and Falicoff, W., "Simultaneous multiple surface optical design method in three dimensions," Opt. Eng. 43, 1489 (2004).

[20] Duerr, F., Benítez, P., Miñano, J. C., Meuret, Y., and Thienpont, H., "Analytic design method for optimal imaging: coupling three ray sets using two free-form lens profiles," Opt. Express 20(5), 5576-5585 (2012).

[21] Duerr, F., Benítez, P., Miñano, J. C., Meuret, Y., and Thienpont, H., "Analytic free-form lens design in 3D: coupling three ray sets using two lens surfaces," Opt. Express 20(10), 10839-10846 (2012).

[22] Grabovičkić, D., Benítez, P., and Miñano, J. C., "Aspheric V-groove reflector design with the SMS method in two dimensions," Opt. Express 18(3), 2515-2521 (2010).

[23] Grabovičkić, D., Benítez, P., and Miñano, J. C., "Free-form V-groove reflector design with the SMS method in three dimensions," Opt. Express 19(104), A747-A756 (2011).

[24] Duerr, F., Benítez, P., Miñano, J. C., Meuret, Y., and Thienpont, H., "Perfect imaging of three object points with only two analytic lens surfaces in two dimensions," Proc. SPIE 8429, 6 (2012). 